\title{
Darboux Transformation in The Tangent-Squared Potential and Supersymmetry
}

\author{
J. Sadeghi ${ }^{1, *}$, A. Pourdarvish ${ }^{2}$, M.Rostami $^{1}$ \\ ${ }^{1}$ Department of Physics, Islamic Azad University - Ayatollah Amoli Branch, P.O.Box 678, Amol, Iran \\ 2 Department of Statistics, Mazandaran University, Babolsar, Iran \\ ${ }^{*}$ Corresponding Author: pouriya@ipm.ir
}

Copyright (C2014 Horizon Research Publishing All rights reserved.

\begin{abstract}
In this paper, we consider one-dimensional Schrödinger equation for the trigonometric tangent-squared potential. Here, we construct the first-order Darboux transformation and the real valued condition of transformed potential for trigonometric tangent-squared potential equation. Also we obtain the transformed of potential and wave function. Finally, we discuss the correspondence between Darboux transformation and supersymmetry. In order to have supersymmetry and commutative and anti-commutative algebra, we obtain some condition for the corresponding equation.
\end{abstract}

Keywords Trigonometric Tangent-Squared Potential; Darboux Transformation; First Order Operators; Supersymmetry

\section{Introduction}

As we know, there are several methods to study for the integrability model. The method we focus here is Darboux transformation. It is well known that the Darboux transformation [1] is one of the major tools for the analysis of physical systems and for finding new solvable systems. Using a linear differential operator , Darboux construct solutions of one ordinary differential equation in terms of another ordinary differential equation. It has been shown that the transformation method is useful in finding soliton solutions of the integrable systems [2-4] and in constructing supersymmetric quantum mechanical systems [5-7]. Also, more general solvable cases were obtained by means of factorization methods [8] and via lie algebraic approaches [9-13]. Darboux transformation is known as one of the most powerful methods for finding solvable Schrödinger equations with constant mass, in the context of which it is also called supersymmetric factorization method [14]. On the other hand during the past few years there has been great interest in studying class of trigonometric potentials [15]. The solution of such equation may be found by mapping it into a Schrödinger - like equation. So, we take advantage from Darboux transformation to this equation and obtain the generalized form of trigonometric tangent - squared equation. The Darboux transformation has been extensively used in quantum mechanics in the search of isospectral potential for exactly Schrödinger equations of constant mass and position - dependent mass [16-21]. So, we take advantage from such transformation and obtain the effective potential, modified wave function and shape invariance condition and generators of supersymmetry algebra for the trigonometric tangent-squared potential. This paper is organized as follows: We first introduce the one-dimensional Schrödinger equation for the trigonometric tangent-squared potential and apply the Darboux transformation to such equation. In that case we show the corresponding potential changes to new form of potential. Finally, we study the supersymmetry version and shape invariance condition for transformed trigonometric tangent-squared potential.

\section{Trigonometric Tangent-Squared Potential.}

Recently, Taseli [22] studied the squared tangent potential $V(x)=\nu(\nu-1) \tan ^{2}(x), x \in\left(\frac{-\pi}{2}, \frac{\pi}{2}\right)$ also known as the symmetric Pöschl-Teller potential [23, 24]. It was noted that the energy eigenvalues, but not the eigenfunctions, of the Hamiltonian with the squared tangent potential on the symmetric interval $\left(\frac{-\pi}{2}, \frac{\pi}{2}\right)$ are precisely the same as those of the Hamiltonian with the squared cotangent potential $V(x)=\nu(\nu-1) \cot ^{2}(x)$ on the asymmetric interval 
$(0, \pi)$ considered by Marmorino in [25] (see also [26]). This observation can be easily verified using the identity

$$
\tan \left(x+\frac{\pi}{2}\right)=-\cot (x)
$$

In this section, we use the asymptotic iteration method to study the Schrödinger equation with the confining potential

$$
V_{x}=V_{0} \tan ^{2}\left(\frac{x}{a}\right), \quad x \in\left(-\frac{\pi a}{2}, \frac{\pi a}{2}\right), \quad V_{0} \geq 0
$$

contained within an infinite square well with sides at $x=\frac{\pi a}{2}$ and $V_{0}$ gives an indication of how rapidly the potential increases within the well. In this case, the time-independent Schrödinger equation reads

$$
\left[-\frac{d^{2}}{d x^{2}}+V_{0} \tan ^{2}\left(\frac{x}{a}\right)\right] \Psi(x)=E \Psi(x), \quad \Psi\left(-\frac{\pi a}{2}\right)=\Psi\left(\frac{\pi a}{2}\right)=0
$$

We, however, take a slightly different approach to solve (3) by letting the wave function assume the form

$$
\Psi(x)=\cos \left(\frac{x}{a}\right) f(x)
$$

we rewrite the equation (3) as,

$$
f^{\prime \prime}(x)=\frac{2}{a} \tan \left(\frac{x}{a}\right) f^{\prime}(x)+\left[\frac{1}{a^{2}}-E+V_{0} \tan ^{2}\left(\frac{x}{a}\right)\right] f(x) .
$$

In order to have a known associated Legndere equation we take $x=a \arcsin (y)$, and one can obtain,

$$
f^{\prime \prime}(y)=\frac{3 y}{1-y^{2}} f^{\prime}(y)+\left(\frac{\omega}{1-y^{2}}+\frac{\mu y^{2}}{\left(1-y^{2}\right)^{2}}\right) f(y),
$$

where $\omega=1-a^{2} E$ and $\mu=a^{2} V_{0}$ and $\alpha=\frac{(\sqrt{\mu+1}-1)}{2}$. Here also we choose suitable variable as,

$$
f(y)=u(y) P(y)
$$

and have,

$$
f^{\prime}(y)=u^{\prime}(y) P(y)+u(y) P^{\prime}(y)
$$

and

$$
f^{\prime \prime}(y)=u^{\prime \prime}(y) P(y)+2 u^{\prime}(y) p^{\prime}(y)+u(y) P^{\prime \prime}(y) .
$$

We substitute equation (8) and (9)into equation(6), we have following equation,

$$
\begin{gathered}
\left(1-y^{2}\right) P^{\prime \prime}(y)+\left[2\left(1-y^{2}\right) \frac{u^{\prime}}{u}-3 y\right] P^{\prime}(y) . \\
+\left[\left(1-y^{2}\right) \frac{u^{\prime \prime}}{u}-3 y \frac{u^{\prime}}{u}-\omega-\frac{\mu y^{2}}{\left(1-y^{2}\right)}\right] P(y)=0 .
\end{gathered}
$$

As we know the associate - Legendre differential equation [27-29] will be as,

$$
\begin{gathered}
\left(1-y^{2}\right) P_{n, m}^{\prime \prime \alpha, \beta}(y)-[\alpha-\beta+(\alpha+\beta+2) y] P_{n, m}^{\prime \alpha, \beta}(y), \\
+\left[n(\alpha+\beta+n+1)-\frac{m(\alpha+\beta+m)+m(\alpha-\beta) y}{1-y^{2}}\right] P_{n, m}^{\alpha, \beta}(y)=0 .
\end{gathered}
$$

Now we compare equations (10) and (11) to each other so one can obtain $u(y)$ as,

$$
2\left(1-y^{2}\right) \frac{u^{\prime}}{u}-3 y=(\beta-\alpha)-(\alpha+\beta+2) y
$$

and

$$
u(y)=\left(\frac{1+y}{1-y}\right)^{\frac{\beta-\alpha}{4}}\left(1-y^{2}\right)^{\frac{\alpha+\beta-1}{4}} .
$$

So, the corresponding wave function will be as,

$$
\begin{gathered}
f(y)=\left(\frac{1+y}{1-y}\right)^{\left(\frac{\beta-\alpha}{4}\right)}\left(1-y^{2}\right)^{\frac{(\alpha+\beta-1)}{4}} P_{n, m}^{(\alpha, \beta)}(y) \quad y=\sin \left(\frac{x}{a}\right), \\
f(x)=\left[\frac{1+\sin \left(\frac{x}{a}\right)}{1-\sin \left(\frac{x}{a}\right)}\right]^{\left(\frac{\beta-\alpha}{4}\right)} \cos \left(\frac{x}{a}\right)^{\frac{(\alpha+\beta-1)}{2}} P_{n, m}^{(\alpha, \beta)}(x)
\end{gathered}
$$


So, we obtain the energy spectrum and wave function as follow,

$$
E=\frac{\hbar^{2}}{2 \mu a^{2}}\left[1-a^{2} V_{0}-(2 m+1) \frac{(\alpha+\beta+3)}{4}+n(\alpha+\beta+n+1)\right]
$$

also

$$
m=-\frac{\alpha+\beta}{2}
$$

and

$$
\Psi(x)=\left[\frac{1+\sin \left(\frac{x}{a}\right)}{1-\sin \left(\frac{x}{a}\right)}\right]^{\left(\frac{\beta-\alpha}{4}\right)} \cos \left(\frac{x}{a}\right)^{\frac{(\alpha+\beta+1)}{2}} P_{n, m}^{(\alpha, \beta)}(x),
$$

where $\alpha$ and $\beta$ are Legendre parameters and will be constant and $n$ and $m$ are degree of such polynomial.

\section{Darboux Transformation and trigonometric tangent-squared poten- tial} by,

As we know the one-dimensional Schrödinger equation for the trigonometric tangent-squared potential is given

$$
f^{\prime \prime}(y)=\frac{3 y}{1-y^{2}} f^{\prime}(y)+\left(\frac{\omega}{1-y^{2}}+\frac{\mu y^{2}}{\left(1-y^{2}\right)^{2}}\right) f(y)
$$

Now we rewrite the trigonometric tangent-squared potential equation (19) as,

$$
\left(1-y^{2}\right) f_{y y}-3 y f_{y}-\left(\omega+\frac{\mu y^{2}}{\left(1-y^{2}\right)}\right) f=0 .
$$

Now we take $F=\left(1-y^{2}\right), G=-3 y$ and the potential $V=\omega+\frac{\mu y^{2}}{\left(1-y^{2}\right)}$. Here we can rewrite the above equation as,

$$
F \partial_{y y}+G \partial_{y}-V=0
$$

and

$$
i C_{t}+\eta C=0, \quad \eta=F \partial_{y y}+G \partial_{y}-V
$$

In order to have same equation as (19) with different of potential. we have to write following equation,

$$
i \hat{C}_{t}+\hat{\eta} C=0, \quad \hat{\eta}=F \partial_{y y}+G \partial_{y}-\hat{V}
$$

Where $V \neq \hat{V}$, this lead us to imply $C \neq \hat{C}$. In order to obtain the modified potential $\hat{V}$ and corresponding wave function for equation (22), we introduce operator $\Delta$ which is called Darboux transformation. The general form of such Durboux transformation will be as,

$$
\Delta=A+B \partial_{y}
$$

The operator $\Delta$ transforms any solution $f(y)$ of (6) into a new solution.

$$
\hat{f}(y)=\Delta f(y)
$$

Where we take special case as $A=B$. In order to find $A$ or $B$, we insert the explicit form of the Hamiltonian $\Delta$ and $\hat{\Delta}$ into the Darboux transformation operator (11) and apply it to the solution $g(y)$ of Eq.(6), we can obtain,

$$
\Delta\left(i \partial_{t}+\eta\right) \Psi(y)=\left(i \partial_{t}+\hat{\eta}\right) \Delta \Psi(y)
$$

Making linear independence of $g(y)$ and its partial derivatives, we collect their respective coefficients and equal them to zero, from which we can obtain the following system about the functions $A$ and $\hat{V}$.

$$
2 F=(F)_{y} B \quad \Rightarrow \quad B=\frac{-1}{y}\left(1-y^{2}\right)
$$

So, the operator Darboux transformation is

$$
\Delta=\frac{-1}{y}\left(1-y^{2}\right)\left(1+\partial_{y}\right) \quad \text { or } \quad \Delta=-\cot \left(\frac{x}{2}\right)\left[\sin \left(\frac{x}{2}\right)-2 \frac{d}{d x}\right]
$$

Now, we achieve the generalized form of wave function which is corresponding to usual wave function $\hat{f}(y)$ as,

$$
\hat{f}(y, t)=\Delta f(y, t)=\frac{-1}{y}\left(1-y^{2}\right)\left(1+\partial_{y}\right) f(y, t), \quad y=\sin \left(\frac{x}{2}\right)
$$




$$
\begin{aligned}
\hat{f}(x, t)= & {\left[-\sin \left(\frac{x}{a}\right) \cot \left(\frac{x}{a}\right)+\frac{\beta-\alpha}{2} \sin \left(\frac{x}{a}\right)+\frac{\alpha+\beta+1}{2}\right] \Psi(x, t)+} \\
& a \cot \left(\frac{x}{a}\right)\left[\frac{1+\sin \left(\frac{x}{a}\right)}{1-\sin \left(\frac{x}{a}\right)}\right]^{\left(\frac{\beta-\alpha}{4}\right)} \cos \left(\frac{x}{a}\right)^{\frac{(\alpha+\beta+1)}{2}} P_{n, m}^{\prime \alpha, \beta}(x)
\end{aligned}
$$

As we know the usual $f(x, t)=e^{-\frac{i E t}{\hbar}} f(x)$ help use to obtain the modified potential, which is given by,

$$
\hat{V}=V+2 \cot ^{2}\left(\frac{x}{a}\right)+6 \tan ^{2}\left(\frac{x}{a}\right)+2 \mu\left(\frac{\tan \left(\frac{x}{a}\right)}{\cos ^{3}\left(\frac{x}{a}\right)}\right)+5
$$

\section{Supersymmetry and Darboux Transformation}

In what follows, we will prove that the formalism of supersymmetry for our generalized trigonometric tangentsquared equation is equivalent to the Darboux transformation. Here we suppose such operator $i \partial_{t}-\theta$ is self-adjoint,

$$
\left(i \partial_{t}+\theta\right)^{\star}=i \partial+\theta
$$

Taking the operation of conjugation on Darboux transformation (23), we obtain

$$
\left(i \partial_{t}+\eta\right) \Delta^{\star}=\Delta^{\star}\left(i \partial_{t}+\hat{\eta}\right)
$$

where the operator $\Delta^{\star}$ adjoint to $\Delta=\alpha\left(1-y^{2}\right)\left(1+\partial_{y}\right)$ is given by,

$$
\Delta^{\star}=\alpha\left(1-y^{2}\right)\left(1-\partial_{y}\right)
$$

Eqs. (21) and (23) can then be rewritten as one single matrix equation of the form,

$$
\left[\begin{array}{cc}
i \partial_{t}+\eta & 0 \\
0 & i \partial_{t}+\hat{\eta}
\end{array}\right]\left[\begin{array}{l}
C \\
\hat{C}
\end{array}\right]=0
$$

Assuming that $H=\operatorname{diag}(\eta, \hat{\eta})$ and $C=(c, \hat{c})^{T}$, the above equation can be written as,

$$
\left[i \partial_{t}+H\right] C=0 .
$$

Two supercharge operator $Q$ and $Q^{\star}$ are defined as follows,

$$
Q=\left[\begin{array}{cc}
0 & 0 \\
\Delta & 0
\end{array}\right], \quad Q^{\star}=\left[\begin{array}{cc}
0 & \Delta^{\star} \\
0 & 0
\end{array}\right],
$$

where $\Delta$ and $\Delta^{\star}$ operators are given by Eqs. (24) and (32), respectively. One can show that the Hamiltonian $H$ satisfies the following expressions

$$
\begin{aligned}
\{Q, Q\} & =\left\{Q^{\star}, Q^{\star}\right\}=0 \\
{\left[Q, i \partial_{t}+H\right] } & =\left[i \partial_{t}+H, Q\right] \\
{\left[Q^{\star}, i \partial_{t}+H\right] } & =\left[i \partial_{t}+H, Q^{\star}\right]
\end{aligned}
$$

Considering the complementing relations of the supersymmetry algebra; the anti commutators $\left\{Q, Q^{\star}\right\}$ and $\left\{Q^{\star}, Q\right\}$, we obtain the operators $R=Q^{\star} Q$ and $\hat{R}=Q Q^{\star}$ and consider the relations between them with our Hamiltonian $\eta$ and $\hat{\eta}$. So, one obtain the $R$ and $\hat{R}$ as follow,

$$
R=|\alpha|^{2}\left[F\left(1-\partial_{y y}\right)-(F)_{y}\left(\partial_{y}+1\right)\right]
$$

and

$$
\hat{R}=|\alpha|^{2}\left[F\left(1-\partial_{y y}\right)-(F)_{y}\left(\partial_{y}+1\right)-\frac{1}{2}(F)_{y y}+\frac{F_{y}}{2 F}\right]
$$

where the indices $y$ will be derivative with respect to $y$. In order to have shape invariance and supersymmetric algebra we need to obtain the $\hat{R}-R$. If such value be constant and zero there is some supersymmetry partner for the system. Otherwise we need to apply some condition in $\hat{R}-R$ to have constant value. So, one can obtain the following equation for the $\hat{R}-R$

$$
\hat{R}-R=|\alpha|^{2}\left(1-\frac{y}{1-y^{2}}\right)
$$

We mention here that if we want the supersymmetry algebra we need to have also the following commutation relation, and also anti-commutation relation between $Q$ and $Q^{+}$.

$$
\left\{Q, Q^{+}\right\}=H, \quad\{Q, Q\}=\left\{Q^{+}, Q^{+}\right\}=0 .
$$

where $Q$ and $Q^{+}$are supercharges.If we look at the equation (39) we need to apply the condition $\hat{R}-R$ to be zero or constant. As we know the limit of $x \in\left[-\frac{\pi a}{2}, \frac{k \pi a}{2}\right]$ we have not shape invariant condition, so we can'nt define supersymmetry. But if we have $x \in\left[-\frac{k \pi a}{2}, \frac{\pi a}{2}\right]$, and $k$ be even we achieve the shape invariance condition and also have supersymmetry charges. For the case of $k$ odd there is not shape invariance. Other case if $\alpha$ ( $\alpha$ is constant), or zero we have supersymmetry system. 


\section{Conclusion}

In this paper we studied the trigonometric tangent-squared equation. We used the first-order Darboux transformation and applied to such system. In order to relate between supersymmetry and Darboux transformation we discussed the supersymmetry algebra and its commutation and anti-commutation superalgebra. We have shown that for satisfying such anti-commutation supercharges the $\hat{R}-R$ must be constat. Also, we applied the condition on the $\hat{R}-R$ and shown that the limits of $x \in\left[-\frac{k \pi a}{2}, \frac{k \pi a}{2}\right]$, there is completely guarantee relation between supersymmetry and Darboux transformation, if $k$ be even number.

\section{REFERENCES}

[1] G. Darboux, Surune proposition relative aux equation lineaires, Compt. Rend. 94 (1882) 1456-1459.

[2] V. B. Matveev and M. A. Salle, Darboux Transformations and Solitons, Springer, Berlin, (1991).

[3] M. J. Ablowitz and H. Segur, Solitons and the Inverse Scattering transform, SIAM, Philadelphia, PA, (1981).

[4] C. H. Gu, H. S. Hu and Z. X. Zhou, Darboux Transformations in Integraable Systems, Mathematical Physics Studies, Vol. 26, Springer, Dordrecht, (2005).

[5] E. Witten, Constraints on supersymmetry breaking, Nucl. Phys. B 202 (1982) 253.

[6] Q. P. Liu and X. B. Hu, Bilinerarization of $N=1$ supersymmetric Korteweg-de Vries equation revisited, J. Phys. A Math. Gen. 38 (2005) 6371-6378.

[7] F. Cooper and A. Khare and U. Sukhatme, Supersymmetry and quntum mechanics, Phys. Rep. 251 (1995) $267-285$.

[8] A. R. Piastino et al, Supersymmetry approach to quantum systems with position - dependent effective mass, Phys. Rev.A 60 (1999) 4318-4325.

[9] R. Kok and M. Koka, A sestematic study on the exact solution of the position dependent mass Schrödinger equation, J. Phys. A 36 (2003) 8105.

[10] J. Sadeghi,Superalgebras for three interacting particles in an external magnetic field, Eur. Phys. J. B 50 (2006)453-457,.

[11] H. Fakhri, and J. Sadeghi,"Supersymmetry approaches tO the bound states of generalized Wood-Saxson potential, Mod. Phys. Lett. A 19 (2004) 615.

[12] J. Sadeghi and M. Rostami, The Supersymmetry Approaches to the Non-central Kratzer Plus Ring-Shaped Potential. IJTP.48: 2961-2970 (2009).

[13] J. Sadeghi,Dirac oscillator with minimal lengths and free particle on AdS2 and S2, J. Math.Phys. 48 (2007)113508.

[14] B. G. Idis, M. M. Musakhanov and M. Sh. Usmanov, Application of supersymmetry and factorization methods to solution f dirac and Schrödinger equations, Theor. Math. Phys. 101 (1984) 1191-1199.

[15] H. Ciftci, R. L. Hall and N. Saad, Exact and approximate of Schrödinger's equation fo a class of trgonometric potentials, arXiv:1210.2467.

[16] Morales J, Pena JJ, Lopez- Bonilla JL. Generalization of the Darboux transform. J Math Phys 2001; 42: 966-75.

[17] Pena JJ, Ovando G, Morales-Guzman D, Morales J, Solvable quartic potentials and their isospectral partners. Int J Quant Chem 2001; 85: 244-50.

[18] Morales J, Pena JJ, Rubio-Ponce A. New Isospectral generalized potentials. Theor Chem Acc 2003; 110: 403-9.

[19] Rubio-Ponce A, Pena JJ, Morales J. One-parameter isospectral solutions for the Fokker-Planck equation. Phys A 2004; 339: 285-95.

[20] Schulze-Halberg A. Effective mass hamiltonians with linear terms in the momentum: Darboux transformations and form-preserving transformations. Int J Mod Phys A 2007;22 : 1735-69.

[21] Schulze-Halberg A. Darboux transformations for effective mass Schrödinger equations with energy-dependent potentials. Int J Mod Phys A 2008;23 : 537-46. 
[22] H. Taseli, J. Math. Chem. 34 (2003) 243.

[23] F.Gori and L. de la Torre, Eur.J. Phys. 24 (2003) 15.

[24] M. M. Nieto and L. M. Simmons, Phys. Rev. D 20 (1979) 1332-1341.

[25] M. G. Marmorino, J. Math. Chem. 32 (2002) 303.

[26] [16] Zhong-Qi Ma, A. Gonzalez-Cisneros, Bo-Wei Xu, and Shi-Hai Dong, Phys. lett. A 371 (2007) 180-184.

[27] F.Cooper, A. Khare, U.Sukhatme: Supersymmetry in Quantum Mechanics. World Scientific, Singapore (2001).

[28] Jafarizadeh, M.A., Fakhri, H.: Parasuppersymmetry and shape invariance in differential equation of mathematical physics and quantum mechanics. Ann. Phys. (NY) 262, 260276 (1998).

[29] Jafarizadeh, M.A., Fakhri, H.: The embedding of parasuppersymmetry and dynamical symmetry into GL(2,c) group. Ann. Phys. (NY) 266, 178206 (1998) 